\title{
THE MANAGEMENT OF ACUTE GENERAL PERITONITIS
}

\author{
By C. R. Savage \\ Resident Assistant Surgeon, St. Thomas's Hospital, London
}

\section{Introduction}

Inflammation of the peritoneal cavity is usually due to bacterial infection which may be preceded in certain instances by an initial chemical peritonitis. Infection most often commences locally in some part of the peritoneal cavity and particularly with early and efficient treatment frequently remains confined to that area. It may, however, become widespread and it is to this condition that the term acute general peritonitis is applied. In many cases when the cause of this infection is removed at an early stage, inflammation although widespread, never becomes established in the peritoneum. This distinction between a general peritonitis which has, and one which has not, become established, although largely a matter of degree is one of great importance as regards treatment. In the former case such measures as the parenteral administration of fluids and the use of gastrointestinal aspiration may well be essential, while in the latter, in the majority of cases, they are quite unnecessary. The causes of general peritonitis are many and varied, but the most common are acute appendicitis and perforations of the stomach and duodenum; a not inconsiderable number of instances occur after elective surgery on the gastrointestinal tract and these carry a very high mortality. There are a number of principles underlying efficient management and these will now be considered.

\section{Early Elimination of the Source of Infection}

In the majority of instances organisms gain access to the peritoneal cavity through perforations in the walls of hollow viscera. Where the source of infection can be dealt with at an early stage either by closure of a perforation, as in perforated peptic ulcer or by removal of the infected organ as in acute appendicitis, the peritoneum is usually well able to overcome any infection which remains. Where, however, a persistent leakage of infected material occurs into the peritoneal cavity either as a result of delay in treatment of an acute abdominal condition or occasionally following an operation on the gastro- intestinal tract, a diffuse and often fatal peritonitis may ensue. Considerable efforts on the part of the body may be made in an attempt to seal off the area of such perforations and these are on occasion successful. Diversion of the intestinal contents from the site of the perforation will certainly assist in this process. It may take the form of gastric aspiration in cases of perforated peptic ulcer or of proximal colostomy above the site of a colonic perforation. It is seldom wise, however, to rely on this measure alone to control continued peritoneal contamination, for in the former instance it may not be possible to keep the stomach completely empty and even if this is successful, duodenal reflux may still occur, while in the latter intestinal contents below the level of the colostomy may still continue to discharge through the perforation. Good results have, however, been obtained in the conservative treatment of perforated gastric and duodenal ulcers by gastric aspiration (Hermon Taylor), but it is considered that early surgical closure is still the treatment of choice except in those cases in which the operative risks are considered to be unduly high. Diversion of the stream of intestinal contents remains a valuable adjuvant measure.

Removal of infected material which has already accumulated in the peritoneal cavity should be carried out at operation with suction and the gentle use of gauze swabs. All methods of irrigation have long since been abandoned because of their disastrous effect of spreading infection and because of the severe shock that they produced. It has long been realized also that attempts to drain the general peritoneal cavity are quite futile owing to the rapid sealing off of the drain track. In addition there are certain positive dangers and disadvantages associated with the use of drainage tubes, such as the formation of adhesions, the erosion of surrounding structures (if the tube is too rigid), the giving of unnecessary pain, and the possible formation of an avenue of infection to the peritoneal cavity from without. Used, however, with certain definite objectives in mind, drainage may be of very great value. Such is the case when 
doubt is felt about the integrity of a suture line, when it is not possible to remove an infected organ or infected material which forms part of an inflammatory mass or the wall of an abscess cavity, or when adequate haemostasis cannot be ensured. In these cases the presence of a drainage tube to the site rapidly forms an exit for infected discharges which might otherwise form a local abscess or spread elsewhere within the peritoneal cavity.

\section{The Assistance of the Natural Processes of the Body in the Resolution and Localization of the Infection}

Widespread infection of the peritoneal cavity may terminate in one of the following ways. It may undergo resolution with complete absorption of the inflammatory exudate with or without the formation of adhesions. It may result in the death of the patient usually by causing a general toxaemia with peripheral circulatory failure due to a combination of factors amongst which continued sepsis, interference with the water, electrolyte and protein balances of the body and paralytic ileus are the most important. It may localize into one or more areas with the formation of abscesses which may in their turn either be absorbed, extend in various directions and discharge either spontaneously or with surgical aid into the lumen of the intestine, externally or, on rare occasions, into the general peritoneal cavity.

It cannot be emphasized too strongly that a most important part of the management of these cases consists in repeated clinical examination to ascertain the presence and site of these residual abscesses and in their efficient treatment. Where the bowel forms part of the wall of the abscess cavity, discharge frequently occurs into the lumen, but when this process is delayed, with deterioration in the general condition of the patient, when the abscess points externally or where anatomical factors preclude this termination (e.g. subphrenic abscess) surgical evacuation is necessary. If for any reason such treatment is delayed for too long, sepsis may spread either locally or widely in the peritoneal cavity with disastrous results. On rare occasions due either to the effect of antibiotics, to high resistance on the part of the individual or to low resistance of the organisms concerned, a chronic septic peritonitis may ensue with multiple plastic adhesions and scattered collections of pus and fibrin between the loops of intestine. In such cases either as the result of leakage of air from the bowel at some unknown site or due to the presence of gas-forming organisms, a form of spontaneous pneumoperitoneum may occur with multiple encysted collections of gas and fluid which on radiography may closely resemble distended segments of bowel. Fig. I shows the X-rays of such a case. An opera- $\frac{2}{3}$ tion for the relief of intestinal obstruction had been $\stackrel{\mathbb{Q}}{\varnothing}$ performed three weeks previously and had been $c$. followed by general peritonitis and paralytic ileus. $\overrightarrow{\vec{F}}$ Three days after the abdomen had returned to normal size following resumption of bowel activity, 응 it again became grossly distended, but this time $\frac{\overline{0}}{\bar{N}}$ with gas and fluid outside the lumen of the bowel. $\frac{\widehat{\sigma}}{\widehat{D}}$ Further operation confirmed these X-ray findings. 을

The process of localization of infection within कै the peritoneal cavity is brought about by the $\vec{\circ}$

FIG. I.-X-rays in erect and horizontal lateral positions $\vec{\omega}$ showing encysted intraperitoneal collections of $\frac{7}{8}$ fluid and gas following acute general peritonitis. (The dense shadows in the horizontal film are due to 3 mercury in the bowel lumen following rupture of iN the balloon of a Miller Abbott tube.)

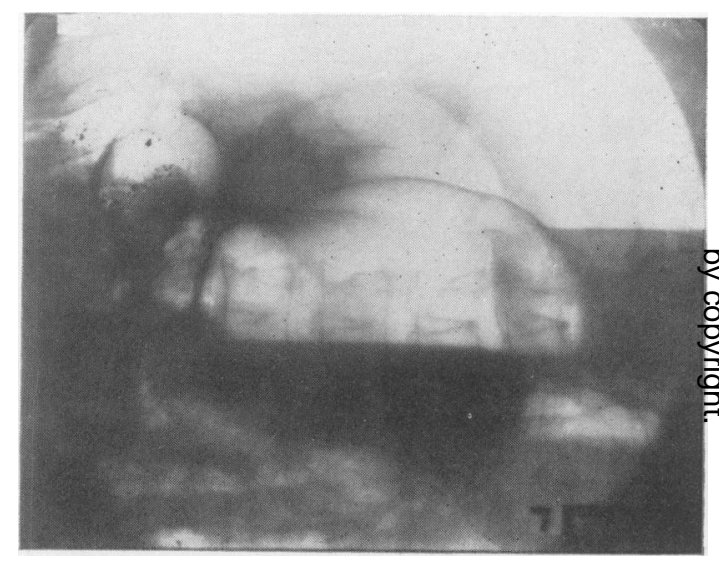

FIg. Ia.-Horizontal lateral X-ray. Spontaneous pneumoperitoneum.

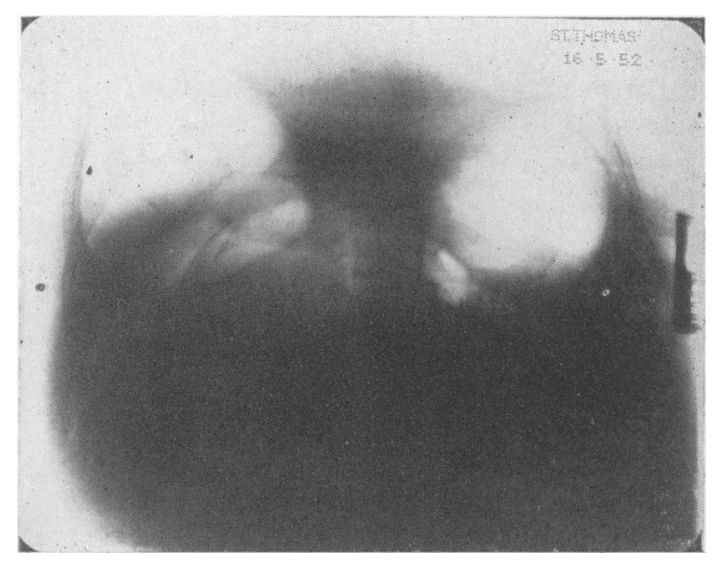

FIG. Ib.-X-ray in erect position. From the same patient as in Fig. ra. 

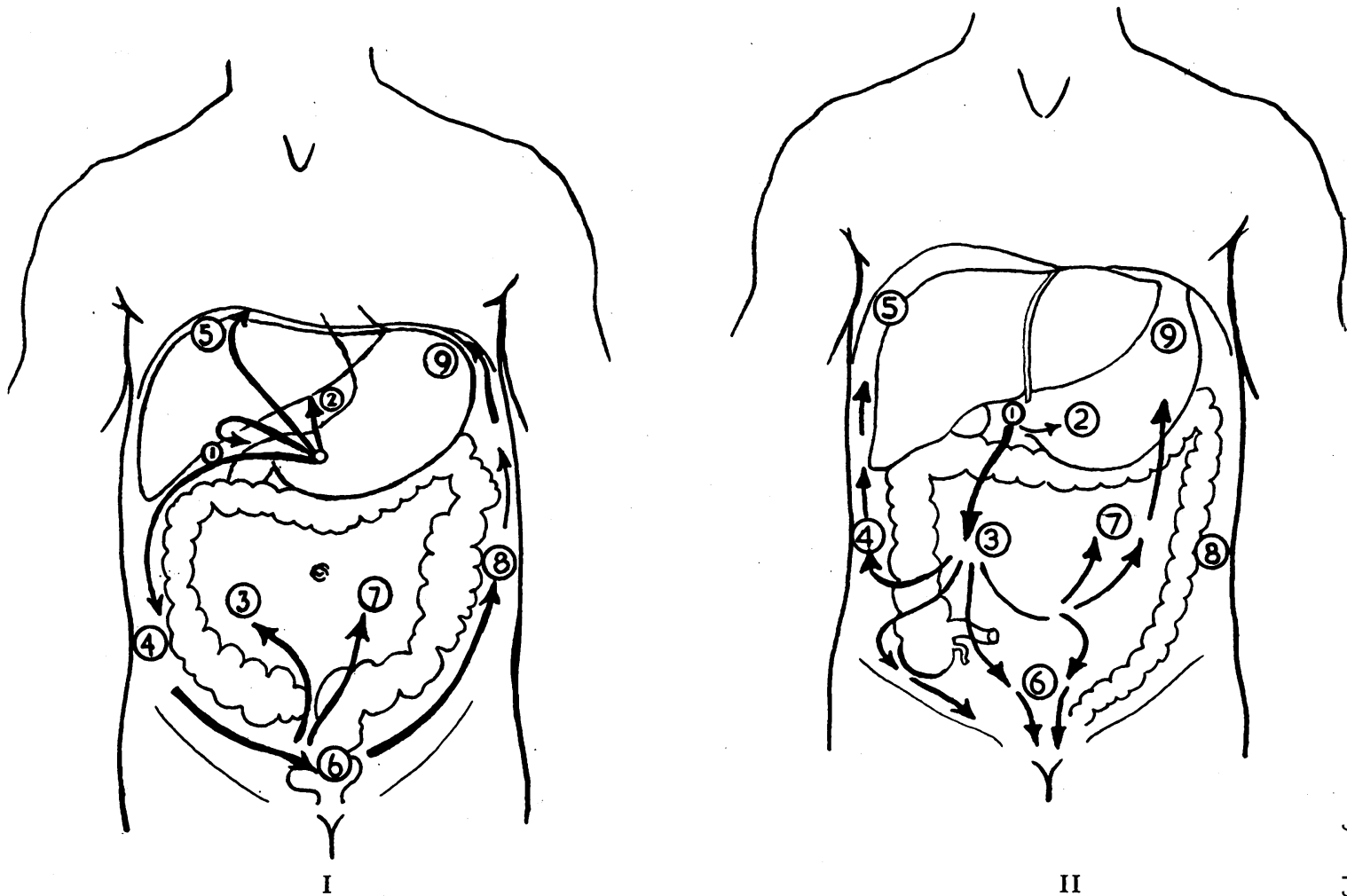

FIG. 2.- Spreading peritonitis: Directions of spread of intraperitoneal fluids following perforations in the region of the pylorus. (I) According to Livingstone, and (II) according to Mitchell. Intraperitoneal spaces are numbered as follows: (1) Subhepatic Space, (2) Lesser Sac, (3) Right Supracolic Space, (4) Right Paracolic Gutter, (5) Right Subphrenic Space, (6) Pelvic Space, (7) Left Supracolic Space, (8) Left Paracolic Gutter, (9) Left Subphrenic Space.

adhesion of the inflamed surfaces of the peritoneum to one another, the omentum playing a conspicuous part, the reflex inhibition of intestinal movements and the reflex rigidity of the abdominal wall. If follows, therefore, that any measure which tends to interfere with this process of localization must be strictly avoided. Manipulations at the time of operation should be confined as far as possible to the area of the source of infection and great care should be taken that naturally formed barriers against the spread of infection are not broken down. Only that which is strictly necessary for the immediate welfare of the patient should be undertaken at the emergency operation. No attempt should be made to obtain intestinal movement either by purgation by enemas or by the use of the cholinergic drugs.

Much attention has been directed to the pathways of spread of free intraperitoneal fluids and also to the influence of the position of the patient in relation to the location of intraperitoneal abscesses following acute peritonitis. Mitchell, in a valuable contribution based on work carried out in infant cadavers in which radio-opaque fluid had been introduced through artificial perforations in the stomach and intestines, has shown that some of the previously accepted avenues of spread of fluids are in fact inaccurate. The accompanying diagrams (Fig. 2) shows both the older views of the directions of spread and those indicated by his experimental work in cases of perforations near the pylorus. Certain other factors such as respiratory and peristaltic movements, movement of the patient as a whole, particularly if violent (e.g. vomiting) and the deflecting action of the naturallyformed barriers against infection may somewhat modify this picture in the living subject.

The influence of gravity on the disposition of free intraperitoneal fluids has been appreciated for many years and was the underlying principle in the treatment of peritonitis by the adoption of Fowler's position. It was claimed that the position definitely reduced the incidence of subphrenic abscess and that it encouraged any possible abscess formation to occur in the pelvis, where it was thought peritoneal absorption of toxic sub- 
stances was less active than in the subdiaphragmatic area. Whereas large collections of fluid will certainly be influenced in this way, it is not necessarily true of smaller ones. Spalding has maintained that the Fowler position so far from discouraging may, in fact, predispose to subphrenic abscess formation. He has emphasized the part played by respiratory movement in promoting a constant flow of a thin film of peritoneal fluid in an upward direction towards the subdiaphragmatic area under normal conditions, where it is absorbed together with any small particles which may be present into the diaphragmatic lymphatics. When air is present within the peritoneal cavity (e.g. after laparotomy) this mechanism ceases to function so efficiently. Under these conditions he considers that, in the sitting position, a pocket of air forms beneath the diaphragm which under the influence of respiratory movement may aspirate infected fluid from the subhepatic pouch (which does not empty in that position) into the subphrenic spaces.

It is certainly quite evident that a number of factors are involved in the localization of infection within the subphrenic area and very difficult to assess which will, in fact, be predominant in any particular instance. In practice, moreover, factors other than the location of residual abscesses will modify opinion as to the correct position for the patient with general peritonitis. The importance of rest, both general and local, has long been realized in the treatment of acute inflammatory conditions, but only more recently has it been appreciated that rest is not synonymous with immobility and that the latter may have certain very serious consequences. Important amongst these are the liability to chest complications and the tendency to peripheral vein thrombosis and pulmonary embolism. These dangers far outweight the doubtful possibilities of influencing the site of abscesses. It is now considered that movement and frequent change of position are of the greatest importance to the patient, and that there is no such thing as a correct or an incorrect position. Constant attention must be-paid to improvement of the pulmonary ventilation, to the expectoration of bronchial secretions and to the prevention of circulatory stasis.

\section{Destruction of Infecting Organisms}

The introduction first of all of the sulphonamide group of drugs and later of the antibiotics has been one of the most important advances in the treatment of peritonitis in recent years. Most cases of peritonitis are due to the combined activities of a number of different types of organisms and many of these organisms are sensitive to one or other of the antibiotics. Failure of the antibiotics to over- come the infection may be due to the presence of insensitive organisms, to persistent reinfection of $z$ the peritoneal cavity, to failure of the drugs to $\stackrel{\mathbb{Q}}{\Omega}$ penetrate into infected and necrotic tissue whose $C$ blood supply has been impaired and occasionally $\overrightarrow{\vec{D}}$ to failure of the patient's normal resources against infection to play their necessary part. In rare instances it may be due to inadequate dosage.

Parenteral administration of antibiotics is $\vec{\nabla}$ normally relied upon and is in most cases satisfactory. Attempts to control infection by oral is administration in cases of persistent reinfection $\overrightarrow{0}$ from the alimentary tract are likely to be un- $\vec{\exists}$ successful owing to the loss of peristaltic activity $\vec{\omega}$ of the intestine and the frequent necessity of $\frac{O}{D}$ gastrointestinal aspiration. Local use within the peritoneal cavity is unwise in view of the formation of foreign body granulomas and adhesions. Owing $\infty$ to their wide range of activity, antibiotics have almost entirely superseded the sulphonamides in the treatment of peritonitis. It seems doubtful if toxins produced by infecting bacteria themselves 은 are of much importance in contributing to the general toxaemia and circulatory failure that may be present. It is more likely that other substances formed as a result of inflammatory destruction of tissue (perhaps of the nature of toxic amino acids) may be responsible by exerting a direcos action on the bowel wall and on the medullar $\vec{\oplus}$ centres. The enormous area of peritoneal surface available for absorption is obviously of importance but it seems doubtful whether this absorbtive capacity differs significantly in the various parts of the peritoreal cavity.

Correction of the Disturbed Water, Electrolyte and Protein Balance of the Body

There is no doubt that a large part of the circulatory collapse which is so marked a feature in the later stages of peritonitis, especially if complicated by paralytic ileus, is dependent upon the losses of water, electrolytes and protein that occur and to an alteration of their distribution within the cellular, extracellular and vascular compartments of the body. These losses may in fact be of enormous dimensions and occur by vomiting or $\frac{9}{5}$ suction from the upper intestinal tract, in some cases by diarrhoea from the lower, from the lungs, from the kidneys and by sweating. In addition large quantities which cannot be, in fact, accurately $\sigma$ measured, are lost to the body in the formation of $N$ inflammatory exudates and into the lumen of $\underset{\omega}{N}$ distended bowel. The normal intake of fluid may in addition be grossly reduced.

The aim in management in peritonitis must therefore be to restore the normal balance of $\stackrel{\mathscr{N}}{?}$ water, electrolytes and protein in the body and to $?$ maintain it once this has been achieved. In many $\underset{\mathbb{D}}{\vec{D}}$ 
early cases where peritonitis has not become established, this may be accomplished by oral intake alone or supplemented by the rectal route. Where, however, infection has advanced beyond this stage as indicated by operative findings, by nausea, or vomiting (excluding post anaesthetic), by abdominal distension and tenderness and by a rising or sustained high pulse rate, dehydration is likely to be more severe and other measures will be necessary. The main method of administration of fluid must now be the intravenous route, and large amounts may be required to begin with. Careful records of the intake and output of fluid must be kept in all cases. Estimation of the exact requirements is not always easy as all the relevant information necessary is never available. In practice, guidance should be obtained from careful clinical examination of the patient for evidence of under- or over-hydration, from study of the fluid balance charts with special reference to the urinary output and by daily estimation of the urinary chlorides. Progress may be checked periodically by appropriate blood examinations. It is most important to realize that at all times the findings of clinical examination are of primary importance and information gained by other means interpreted strictly in relation to these.

The importance of protein deficiency which may result from loss into inflammatory exudates, into the lumen of the distended intestine, into the intestinal wall and from diminished intake has been emphasized by Rob, who recommended the administration of plasma, sometimes in large quantities, for the prevention and treatment of peripheral circulatory failure in all cases of peritonitis of sufficient severity to require intravenous fluids.

Recent work suggests that potassium loss is also important and any defiiciency must be rectified with due regard to the dangers of over-dosage.

\section{Control of Gastrointestinal Distension}

Reflex depression of gastrointestinal activity of varying degree follows every laparotomy and may, in fact, be initiated reflexly on rare occasions by trauma to more distant parts of the body. The depression of activity is accompanied by a disorganization of the gas and fluid balancing mechanisms of the intestine. In the majority of cases it is of a temporary nature only, but in certain instances a self-perpetuating vicious circle of events is set up and paralytic ileus becomes established. This is particularly likely to occur in diffuse peritonitis. Careful and repeated clinical examination is necessary if the early symptoms and signs of the onset of the condition are to be recognized. These are increasing abdominal distension, absent or altered bowel sounds, nausea and vomiting and a rapid pulse rate. In most cases there is no bowel movement and no flatus is passed per rectum. It is important, however, 'to realize that nausea and vomiting may on occasions be entirely absent, that distension may pass unnoticed.in the lax abdomen (e.g. after parturition or in the obese) and that the only warning signs may then be an otherwise unexplained rising pulse rate in an anxious ill-looking patient. Symptoms of peripheral circulatory failure may follow rapidly. Paralytic ileus may affect either the whole or only a part of the gastro-intestinal tract and diarrhoea may occur in the presence of gross distension particularly in cases where infection is most marked in the pelvis. When in these cases the diarrhoea is accompanied by a gradually rising pulse rate, it appears to be of very ominous significance. The most important causative factors appear to be the failure of the normal gas and fluid balancing mechanisms of the bowel, the disorganization of the propulsive element of peristaltic activity, toxic paralysis of the bowel wall, circulatory stasis and hypoproteinemia. The fluid in the distended bowel is derived from intake by mouth, gastric, intestinal, biliary and pancreatic secretions, and fluid diffused from the blood through the oedematous inflamed and ill-nourished bowel wall. According to Wangensteen the gas is derived largely from swallowed air, the normal absorptive mechanism having broken down and, to a much lesser extent, from diffusion from the blood and from digestive processes.

Management should be directed to the relief of distension and to the maintenance of a satisfactory circulation to the distended bowel as only if this is ensured can there be hope of resumption of normal activity. Gastrointestinal aspiration has been of the greatest value in achieving these objects. Introduced originally for diagnostic purposes its therapeutic possibilities were soon realized. The Miller Abbott double lumen tube, acting as a progressing internal enterostomy, was an additional advance on this principle. Difficulty in introduction of the end of this tube into the duodenum despite careful attention to detail is not infrequent, particularly in those cases in which it might be expected to be of most benefit. Once past the pylorous, however, its progress is usually most satisfactory. It is fortunate that in the majority of cases gastric intubation appears to be efficient in preventing vomiting and in ensuring adequate decompression. It is certainly seldom advisable to persist in repeated attempts to pass the Miller Abbott tube into the duodenum in a 'seriously ill patient. Even when this tube has passed into the small intestine it may on occasion fail to function satisfactorily even when its patency is undoubted. It scems probable that this may be 
due either to the necessarily small calibre of the tube in relation to thick intestinal contents sometimes encountered, to further distention occurring in the bowel above the level of the end of the tube, as suggested by the increased aspirate which may be obtained on withdrawing the tube to a higher level or to the arrest of the tube above an angulated distended loop of bowel. This additional factor of mechanical superimposed upon functional obstruction, caused by angulation of distended bowel is very important and arises in all cases in which distension becomes severe. Multiple points of obstruction are produced and attempted surgical relief is never indicated as these angulations rapidly become reduced once normal peristaltic activity is resumed. Obstruction by adhesions at a single site certainly occurs following peritonitis, but it is seldom seen in the early post-operative period. At one time it was considered that, in some cases, obstruction might be due to a localized loop of small intestine becoming paralyzed by immersion in a collection of pus in the pelvis (the ileus duplex of Sampson Handley), but it seems doubtful if this is ever really so although the relief of obstruction which may follow the drainage of an intraperitoneal abscess tends to support it. It may be, in fact, that the intestine has become angulated and oedematous by becoming involved in the formation of the wall of the abscess and that this is relieved when the pus has been evacuated.

Prolonged inhalation of oxygen in high concentrations has been advocated in an attempt to reduce distension by displacing the nitrogen in the bowel. It is very difficult to judge clinically of its efficacy, but this does not seem to be very great, certainly not sufficient to justify the additional discomfort and manipulation that it entails.

Stimulation of the bowel by various methods has also been advised on many occasions in the treatment of paralytic ileus, but as far as that complicating peritonitis is concerned it is contraindicated. There is no convincing evidence that enemas can induce reflex peristalsis in the small intestine and they may, in fact, add to distension by being retained and fail to relieve even large bowel ileus when this is present. Cholinergic drugs may cause contraction of the bowel but this contraction does not seem to be of the co-ordinated propulsive type necessary for the forward trans- mission of the intestinal contents. They may, in fact, stimulate further secretion from the bowel $\frac{2}{3}$ wall and where the mechanical obstructive element $\mathbb{D}$ has supervened there are other possible dangers...

Certain other factors which have already been dealt with, such as the control of infection and of the water, electrolyte and protein balance of the body will have a direct effect on improving the circulation of the bowel wall and maintaining it in such a condition that its normal function may return. They constitute an essential part of treatment.

\section{Provision of Rest and Relief of Pain}

The importance of ensuring adequate rest in the form of sleep and relief of pain and anxiety in general peritonitis has already been mentioned, and this is even more true when paralytic ileus has $\infty$ ensued. Many conflicting accounts have been given of the effects of morphine on the motility of the alimentary tract. Even if it were proved conclusively, however, that it had inhibitory effects on the intestinal muscle, its enormously valuable general effects would continue to justify its use.

\section{Conclusion}

Improvement in the prognosis of genera peritonitis has been very marked in recent yearso The most important factors responsible are the introduction of the antibiotics, the fuller under $\mathrm{N}$ standing of the fluid, electrolyte and protein balances of the body, the use of gastrointestinal decompression and the earlier diagnosis and treatment of the acute abdomen. More powerful antibiotics with an increased range of activity may become available in the future, but in the absence of the most careful pre- and post-operative management, attention to general surgical principles and to the details of surgical technique, it is doubtful if the outlook in this condition can be further improved to any great extent.

\section{BIBLIOGRAPHY}

TAYLOR, HERMON (1946), Lancet, ii, 44I.

ROB, C. G. (1947), Proc. Roy. Soc. Med., 40, 123

SPALDING, J. E. (1946), Lancet, i, 643.

MITCHELL, G. A. G. (1941), B.F.S., 28, $29 \mathrm{I}$. WANGENSTEEN, O. H. (1942), Int. Obs. Springfield: Charles
C. Thomas. 2nd Edition.

LIVINGSTONE, E. M. (1932), 'A Clinical Study of Abdominal Cavity and Peritoneum ' (Hoeber's Surgical Monographs No. I), New York: Paul B. Hoeber, Inc.

\section{CORRECTION}

We must apologise for the Printer's error which appears on p. 583 of the November issue, this acknowledgment should read (Figs. 2, 3, 5 and 6 reproduced by kind permission of The Medical Press). 